This item was submitted to Loughborough's Research Repository by the author.

Items in Figshare are protected by copyright, with all rights reserved, unless otherwise indicated.

\title{
Life-cycle maintenance of deteriorating structures by multi-objective optimization involving reliability, risk, availability, hazard and cost
}

\section{PLEASE CITE THE PUBLISHED VERSION}

http://dx.doi.org/10.1016/j.strusafe.2014.02.002

\section{PUBLISHER}

(C) Elsevier

\section{VERSION}

AM (Accepted Manuscript)

\section{PUBLISHER STATEMENT}

This work is made available according to the conditions of the Creative Commons Attribution-NonCommercialNoDerivatives 4.0 International (CC BY-NC-ND 4.0) licence. Full details of this licence are available at: https://creativecommons.org/licenses/by-nc-nd/4.0/

\section{LICENCE}

CC BY-NC-ND 4.0

\section{REPOSITORY RECORD}

Barone, Giorgio, and Dan M. Frangopol. 2014. "Life-cycle Maintenance of Deteriorating Structures by Multiobjective Optimization Involving Reliability, Risk, Availability, Hazard and Cost”. Loughborough University. https://hdl.handle.net/2134/16762. 


\title{
Life-cycle maintenance of deteriorating structures by multi-objective optimization involving reliability, risk, availability, hazard and cost
}

\author{
Giorgio Barone ${ }^{1}$ and Dan M. Frangopol ${ }^{2 *}$
}

\begin{abstract}
In recent years, several probabilistic methods for assessing the performance of structural systems have been proposed. These methods take into account uncertainties associated with material properties, structural deterioration, and increasing loads over time, among others. When aging phenomena have significant effects on the life-cycle performance of the structure, it becomes essential to perform actions to maintain or improve structural safety, in agreement with the system requirements and available funds. Various optimization methods and performance indicators have been proposed for the determination of optimal maintenance plans for simple and complex systems. The aim of this paper is twofold: (a) to assess and compare advantages and drawbacks of four different performance indicators related to multiobjective optimization of maintenance schedules of deteriorating structures, and (b) to assess the cost-efficiency of the associated optimal solutions. Two annual performance indicators, annual reliability index and annual risk, and two lifetime performance indicators (i.e. availability and hazard functions) are used in conjunction with total maintenance cost for evaluating Pareto fronts associated with optimal maintenance schedules of deteriorating structures. Essential maintenance actions are considered and optimization is performed by using genetic algorithms. The approach is illustrated on an existing deteriorating bridge superstructure.
\end{abstract}

Author keywords: reliability; risk; availability; hazard; life-cycle maintenance optimization.

\footnotetext{
${ }^{1}$ Research Associate, Department of Civil and Environmental Engineering, Engineering Research Center for Advanced Technology for Large Structural Systems (ATLSS Center), Lehigh University, 117 ATLSS Dr., Bethlehem, PA 18015-4729, USA, gib212@lehigh.edu.

${ }^{2}$ Professor and the Fazlur R. Khan Endowed Chair of Structural Engineering and Architecture, Department of Civil and Environmental Engineering, Engineering Research Center for Advanced Technology for Large Structural Systems (ATLSS Center), Lehigh University, 117 ATLSS Dr., Bethlehem, PA 18015-4729, USA, dan.frangopol@lehigh.edu, *Corresponding Author.
} 


\section{Introduction}

Decision-making problems associated with the optimal maintenance of civil and marine structures and infrastructures are a crucial research topic in the field of life-cycle structural engineering. The increasing number of structural systems reaching critical conditions, due to increasing demands and/or deterioration of the component resistances, has directed researchers' attention towards the development of methods for the determination of costeffective maintenance strategies. Optimization algorithms, having prescribed goals and considering maintenance times as design variables, allow the identification of several possible optimal maintenance strategies during the system life-cycle. The most appropriate intervention can be chosen with respect to several constraints, such as available funds.

Maintenance actions can be preventive, aiming at arresting or slowing down the structural deterioration, or essential, totally or partially restoring the performance of single or multiple components of the system. These actions can be applied at prescribed regular time intervals. However, it has been shown [Frangopol et al. 1997] that non-uniform time intervals are more efficient for maximizing the structural performance over the life-cycle of the system while simultaneously minimizing the total cost of the maintenance plan.

A crucial task for the determination of optimal maintenance plans is to accurately model the system, as well as the stressors and loads acting on it during its entire life-cycle. Probabilistic approaches constitute the most reasonable way to deal with the various uncertainties inherent to this task. Several indicators have been proposed during recent years to represent the time-dependent structural performance of deteriorating structures [Wen and Kang 2001, Ang and De Leon 2005, Ellingwood 2005, Moan 2005, Frangopol 2011]

Two classes of indicators can be easily distinguished. The first includes point-in-time performance indicators, such as annual reliability index, annual risk, redundancy, robustness, and vulnerability [Saydam and Frangopol 2011]. The second class consists of the lifetime distributions, such as survivor, availability, and hazard functions [Yang et al. 2006a]. While some of these indicators have been extensively used in literature, the advantages and 
drawbacks related to their use into optimization frameworks for the determination of optimal maintenance plans have not been specifically addressed.

This paper aims at investigating four of the most commonly used performance indicators, namely annual reliability index, annual risk, availability and hazard functions, for determining optimal maintenance plans for deteriorating structures. A preliminary investigation has been conducted in [Barone and Frangopol 2014] by considering a thresholdbased approach. Instead, bi-objective optimization is considered herein. For each optimization problem, minimizing the total cost of the maintenance plan is considered as the first objective, while the second objective includes minimizing one of the above mentioned performance indicators. Essential maintenance of single or multiple components of a deteriorating system is considered, entailing total restoration of the performance of components to its original value. The components with the highest repair priority are determined differently for each performance indicator, considering minimum reliability, maximum risk, and availability and hazard importance factors.

The approach is illustrated on an existing bridge superstructure modeled as a seriesparallel system whose components are the bridge deck and girders. Pareto fronts and optimal solutions obtained from the four different approaches are compared.

\section{Life-cycle maintenance optimization with different performance indicators}

Life-cycle maintenance of a structural system is a fundamental requirement for maintaining the performance above safety thresholds. A comprehensive maintenance framework should include inspections and maintenance interventions. Inspections can be used to identify structural properties at various stages during the system life-cycle, assess the structural performance and, possibly, update the structural models established in the design phases. Maintenance interventions are, instead, needed to maintain, improve, or restore the system performance. Maintenance actions can be preventive or essential. Preventive maintenance is applied before reaching critical conditions and it is used to stop or delay the structural deterioration processes for a period of time. Typical examples of preventive maintenance are painting and coating of steel girders for corrosion prevention. Essential maintenance is, 
instead, required when the structure has reached prescribed performance thresholds, threatening the system safety. Essential maintenance actions provide a recovery of the structural performance of one or more components that may be partial (e.g., repair of structural components) or total (e.g., replacement of structural components). Since preventive maintenance can be performed when the actual structural deterioration is not critical for the safety of the structure, it is usually applied at regular time intervals over the system life-cycle. On the other hand, to maximize its cost-efficiency, essential maintenance has to be performed at optimal times, before the system failure occurs.

Therefore, life-cycle maintenance should be formulated as an optimization problem with design variables describing the number of repairs and their optimal application times. This optimization can be performed with respect to one or more prescribed performance indicators, considering constraints relative to maintenance costs and to repair times (e.g., minimum time interval between maintenance actions, maintenance effectiveness, among others). An alternative is to use multi-objective optimization techniques considering performance indicators and the maintenance cost as objectives. In this case, the result of the optimization is a set of optimal solutions (i.e. Pareto solutions). Subsequently, the most appropriate solution can be identified.

At each repair time, prescribed components must be repaired. Therefore, it is necessary to establish criteria for selecting which component(s) should be repaired at each intervention time. These criteria have to be defined depending on the performance indicator selected for the life-cycle maintenance optimization problem; in addition, they should be able to assign repair priority to components with the highest impact on the system condition.

A formal multi-objective framework to optimize the lifetime maintenance of deteriorating structures involving reliability, risk, availability, hazard and cost is presented herein by considering an existing deteriorating bridge superstructure.

\section{Maintenance optimization of a deteriorating bridge superstructure}

Four different optimization problems will be discussed in this paper for the determination of the optimal maintenance planning of the superstructure of the E-17-HS bridge located in 
Colorado, over an Interstate Highway. The bridge failure modes, considered in the following sections for computational purposes, are briefly described herein; further details can be found in [Akgül 2002, Marsh and Frangopol 2008]. For illustrative purposes, the reinforced concrete end span of the bridge, whose cross-section is shown in Figure 1(a), has been modeled as a series-parallel system so that the failure occurs when either the deck or two consecutive girders fail (Figure 1(b)). Material properties and bridge resistances and loads are modeled following the data provided in [Akgül 2002]. In particular, for the bridge deck, the limit state function associated with bending is:

$$
g_{0}=K_{1} A_{d} f_{y, d} \lambda_{d} \gamma_{d}-K_{2} \frac{A_{d}^{2} f_{y, d}^{2} \gamma_{d}}{f_{c, d}}-K_{3} \lambda_{\text {trk }}=0
$$

where the cross-sectional area of the deck steel reinforcement $A_{d}$, the associated yield strength $f_{y, d}$, the compressive strength of the concrete $f_{c, d}$, the reinforcement depth uncertainty factor $\lambda_{d}$, the modeling uncertainty factor $\gamma_{d}$, and the effect of the load $\lambda_{\text {trk }}$ due to a HS20 truck are modeled as lognormal random variables. The deterministic coefficients $K_{1}, \ldots, K_{3}$ assume the following values: $K_{1}=4.323 \times 10^{-1}, K_{2}=4.085 \times 10^{-3}, K_{3}=5.287$. Additionally, the most critical failure mode for the girders is associated with the shea; therefore, the following limit state has been considered:

$$
g_{i}=K_{4} \sqrt{f_{c, g}} \lambda_{d, i} \gamma_{g}+K_{5} A_{g, i} f_{y, g} \lambda_{d, i} \gamma_{g}-V_{t r k} I_{f} D_{f}=0
$$

The cross-sectional area of the shear reinforcement in each girder $A_{g, i}$, the associated yield strength $f_{y, g}$, the compressive strength of the girder concrete $f_{c, g}$, the uncertainty factor related to the depth of the reinforcement $\lambda_{d, i}$, the modeling uncertainty factor $\gamma_{g}$, the shear load $V_{t r k}$, the girders impact factor $I_{f}$, and the distribution factor $D_{f}$ have been considered lognormal distributed. The deterministic coefficients $K_{4}$ and $K_{5}$ assume the following values: $K_{4}=30.925, K_{5}=5.093$. The means and standard deviations of the variables considered into Eqs. (1) and (2) are defined in [Akgül 2002]. 
A continuous reduction over time of the cross-sectional area of the reinforcement bars in the bridge superstructure, due to chloride contamination, is considered over the life-cycle of the structure. Loads acting on the bridge are due to the average daily traffic. Readers are referred to [Akgül 2002] for numerical details regarding both the corrosion model and loads. Failure probability for the bridge superstructure, as well as failure probabilities associated with each component (deck, exterior and interior girders) have been evaluated by the First Order Reliability Method (FORM) using the RELSYS software [Estes and Frangopol 1998]. The results are shown in Figure 1(c).

Optimal maintenance plans are investigated for the superstructure of the E-17-HS bridge, based on four different approaches. All the maintenance plans entail essential maintenance actions on the system components, and either deck or girders are considered "as new” after repair. The design variables for each optimization procedure are the repair times; thus each solution of the optimization problem is a sequence of maintenance actions performed on selected components of the bridge.

\section{Bi-objective optimizations based on annual performance indicators and maintenance cost}

Annual performance indicators assume numerical values representing the performance state of a system or its components at a given point-in-time during its life-cycle, computed at one year time intervals. Generally, the system performance is evaluated with respect to selected limit states (e.g., yielding, failure, or serviceability), depending on the aim of the performed analysis, and on the function and strategic importance of the structure. Since several uncertainties are associated with the resistance state of the structural components over time and with the acting loads, annual performance indicators provide a measure of the probability of the selected limit state being reached in a selected year.

Approximate numerical methods can be used for estimating the probability of reaching the selected limit states. The most common among these methods are FORM and the Second Order Reliability Method (SORM). These methods can take into account the random variables associated with the component resistances and loads, the system 
configuration (series, parallel, series-parallel), and the correlation among failure modes. When the analysis is performed over a large temporal window, the computational procedure has to be repeated at each year by considering the resistance and load variation over time, due to structural deterioration phenomena or increasing demand or maintenance actions.

While several annual performance indicators have been defined in literature, annual reliability and annual risk will be studied herein. The former is considered because of its straightforward definition in terms of failure probability and ease of use, and the latter due to its increasing importance in the research field during recent years.

\section{Annual reliability index and total maintenance cost}

The first approach discussed herein is based on the use of the annual reliability index $\beta(t)$. In this section, a bi-objective optimization procedure is considered for optimal maintenance planning of multi-component systems, and a numerical application dealing with the previously introduced series-parallel model of bridge superstructure is proposed. The two goals of the optimization are (a) to maximize the minimum system annual reliability index over a $t_{\text {life }}=75$ years life-cycle, and (b) to minimize the total cost of the maintenance plan. The design variables are the repair times, considering as constraints a six year minimum interval between two subsequent repair actions and no repair after 66 years from the initial time. The system and components reliability profiles are shown in Figure 2(a) for the case of no repair actions. Values of system and components annual reliability index have been obtained by using RELSYS software.

Repair actions are performed on the components with the lowest reliability at the repair time. Maintenance costs for the deck and girders are assumed $\$ 100,000$ and $\$ 40,000$, respectively, and an annual discount rate of money $r=0.02$ is considered. Therefore, the total cost of the maintenance plan $C_{\text {tot }}$ is calculated as:

$$
C_{\text {tot }}=\sum_{i=1}^{N_{\text {comp }}} \sum_{j=1}^{N_{\text {rep }}} \frac{C_{i} \delta_{i j}}{(1+r)^{t_{j}}}
$$


where $C_{i}$ is the cost of the $i$-th component, $N_{\text {comp }}$ is the total number of components, $N_{\text {rep }}$ is the total number of repair actions in the maintenance plan, and $\delta_{i j}=1$ if the $i$-th component is repaired at the year $t_{j}$ while $\delta_{i j}=0$ in the opposite case.

The optimization formulation is described by the following minimization problem:

Given: $g_{i}(t), C_{i}, r \quad i=0, \ldots, 4$

Find: $N_{\text {rep }}, \mathbf{t}_{\text {rep }}=\left\{t_{1}, \ldots, t_{N_{\text {rep }}}\right\}$

To minimize: $\left\{\begin{array}{l}\left(\beta_{\min }(t)\right)^{-1} \\ C_{\text {tot }}\end{array} \quad 0 \leq t \leq 75\right.$ years

Such that: $\left\{\begin{array}{l}t_{j}-t_{j-1} \geq 6 \text { years } \\ t_{N_{\text {rep }}} \leq 66 \text { years }\end{array} \quad j=1, \ldots, N_{\text {rep }}\right.$

where $g_{i}(t)$ are the limit state functions associated with the structural failure modes, $C_{i}$ is the cost of repair of the system components, $r$ is the annual money discount rate, $N_{\text {rep }}$ is the total number of repairs, $\mathbf{t}_{\text {rep }}=\left\{t_{1}, \ldots, t_{N_{\text {rep }}}\right\}$ is the vector of the repair times, $\beta_{\text {min }}(t)$ is the minimum value of the system reliability over its life-cycle, and $C_{\text {tot }}$ is the total cost of the maintenance plan, evaluated by Eq. (3). The first goal in Eq. (6) has been defined in terms of the inverse of the annual reliability index, so that a minimization problem is obtained with respect to both objectives.

The Pareto front of optimal solutions is obtained by using the elitist genetic algorithm provided with MATLAB 7.12 (2011), that is a variant of the algorithm NSGA-II [Deb 2001]. Single point cross-over has been adopted, using an initial population of 300 trial solutions. Mutation and cross-over functions have been modified to avoid non-integer solutions and to comply with constraints defined by Eq. (7). Moreover, to improve the computational efficiency of the procedure, a bookkeeping technique has been implemented [Bocchini and Frangopol 2011] by keeping track of solutions evaluated in previous iterations and avoiding 
redundant calculations. Since the number of repair actions $N_{\text {rep }}$ is variable, the obtained Pareto front is the envelope of the Pareto fronts that are obtained by performing a set of optimizations fixing a priori $N_{\text {rep }}=0, \ldots, 11$ (since 11 is the maximum number of repair actions achievable complying with the constraints in Eq. (7)). However, using a dynamic number of variables allows to run a single optimization routine, instead of dealing with 10 different optimization procedures (i.e. one for each number of repair; the two particular cases of zero and 11 repairs admit only one solution, therefore no optimization is needed in those cases).

The obtained Pareto front is shown in Figure 2(b) together with a subset of nondominant solutions; discontinuities in the Pareto front occur when the number of repair $N_{\text {rep }}$ changes. Figure 2(c) illustrate annual reliability index of the solution denoted as A1 in the Pareto front (Figure 2(b)). Solution A1 is characterized by two repair actions $\left(N_{\text {rep }}^{A 1}=2\right)$, occurring at 45 and 51 years. The maintenance plan involves essential maintenance of the exterior girders first, and subsequently of the deck, since they are, respectively, the components with the lowest reliability at the maintenance times. Repair of the deck provides the highest effect on the recovery of the system reliability. By considering Eq. (3), the cost of the maintenance plan is estimated as $\$ 69,240$. The reliability profile associated with solution A2 in Figure 2(b) is, instead, shown in Figure 2(d). In this case, five repair actions are provided: exterior girders are repaired at 21 and 52 years, interior ones at 45 years, and the deck at 31 and 60 years. In this case, the total cost of the maintenance plan is $\$ 198,770$.

\section{Annual risk and total maintenance cost}

The annual probability of failure and annual reliability index do not contain any information on the consequences associated with the system or component failure. However, annual risk provides additional information, particularly significant when dealing with decision-making processes concerning system performance. Risk for determining optimal maintenance plans for bridges subjected to earthquakes and traffic loads has been proposed by [Zhu and Frangopol 2013], where single-objective minimization is performed for evaluating optimal essential and preventive maintenance schedules. 
Risk is defined as the product of the failure probability and the consequences in monetary terms due to the failure itself [Ang and Tang 1984]. In general, for each component, direct and indirect risks can be distinguished. The former is obtained by considering the direct consequences of failure, usually associated with repair/replacement cost of the component itself. The latter entails an estimation of indirect consequences deriving from failure and not strictly related to rebuilding the structure itself. Indirect consequences may include, for example, injuries, fatalities, or environmental contamination due to the system failure. With respect to the $i$-th component of the system, direct and indirect risks are defined as:

$$
\begin{aligned}
& R_{i}^{\text {dir }}(t)=P\left(F_{i} \bar{F}_{j \neq i}\right) C_{i}^{\text {dir }}(t) \\
& R_{i}^{\text {ind }}(t)=P\left(F_{i} \bar{F}_{j \neq i}\right) P\left(F_{s y s} \mid F_{i} \bar{F}_{j \neq i}\right) C^{\text {ind }}(t)
\end{aligned}
$$

where $F_{i}$ and $\bar{F}_{j}$ denote failure of component $i$ and survival of component $j$, respectively, $P\left(F_{i} \bar{F}_{j \neq i}\right)$ is the failure probability of the component $i$ and survival of all the other components, and $P\left(F_{s y s} \mid F_{i} \bar{F}_{j \neq i}\right)$ is the failure probability of the system without the $i$-th component conditioned by the event $F_{i} \bar{F}_{j \neq i}$. In the following, direct consequences associated with failure of deck and girders of the bridge superstructure are considered equal to their repair costs, defined previously.

Indirect consequences are estimated as the sum of three different losses due to the system failure. The first one is the cost of running vehicles along the detour imposed on the drivers during the bridge maintenance [Stein et al. 1999], defined as:

$$
C_{r}=C_{v} L_{d} A_{D T} d_{d}
$$

where $C_{v}$ is the average running cost of a vehicle $(\$ 0.16 / \mathrm{km}), L_{d}$ is the detour length (assumed $10 \mathrm{~km}$ ), $A_{D T}$ is the average daily traffic (assumed 400 vehicles per day), and $d_{d}$ is the number of days when the bridge, due to maintenance operations, is not accessible (assumed 365 days). 
The second loss considered is the cost of time spent by passengers and trucks driving along the detour, estimated as [Stein et al. 1999]:

$$
C_{t}=\left[C_{a d} O_{c a r}\left(1-\frac{T_{p}}{100}\right)+C_{t r k} \frac{T_{p}}{100}\right] \frac{L_{d} A_{D T} d_{d}}{s}
$$

where $C_{a d}$ and $C_{t r k}$ are the time costs for adult persons (\$7.05/hour) and trucks (\$20.56/hour) respectively, $O_{\text {car }}$ is the average vehicle occupancy in cars (1.56 adult/vehicle), $T_{p}$ is the trucks percentage over the total number of vehicles crossing normally the bridge (assumed $4 \%)$, and $s$ is the average speed along the detour $(64 \mathrm{~km} / \mathrm{h})$.

The last contribution to indirect consequences represents safety and environmental losses assumed as $C_{s}=\$ 5 \mathrm{M}$. Finally, taking into consideration the discount rate of money $r$, direct and indirect consequences are evaluated as:

$$
\begin{aligned}
C_{i}^{\text {dir }}(t) & =\frac{C_{i}}{(1+r)^{t}} \\
C^{\text {ind }}(t) & =\frac{C_{r}+C_{t}+C_{s}}{(1+r)^{t}}
\end{aligned}
$$

The total annual system risk (i.e. the sum of direct and indirect risks) for the bridge superstructure when no repair actions are considered is shown in Figure 3(a) together with the risk associated with each component.

Analogously to what has been used for the reliability case in the previous section, a bi-objective optimization procedure is proposed for determining the optimal maintenance plan for the bridge superstructure considering the same constraints used in the reliabilitybased approach. However, in this case, repair is always performed on the component having the highest associated risk. Moreover, minimization of the maximum system risk is considered as a second goal. Therefore, the optimization problem is as follows:

Given: $g_{i}(t), C_{i}, r \quad i=0, \ldots, 4$ 
Find: $N_{\text {rep }}, \mathbf{t}_{\text {rep }}=\left\{t_{1}, \ldots, t_{N_{\text {rep }}}\right\}$

To minimize: $\left\{\begin{array}{l}R_{\max }(t) \\ C_{\text {tot }}\end{array} \quad 0 \leq t \leq 75\right.$ years

Such that: $\left\{\begin{array}{l}t_{j}-t_{j-1} \geq 6 \text { years } \\ t_{N_{\text {rep }}} \leq 66 \text { years }\end{array} \quad j=1, \ldots, N_{\text {rep }}\right.$

where $R_{\max }(t)$ is the maximum value of the system annual risk over the considered life-cycle span. Also in this case, the number of design variables changes dynamically during the optimization routine, and a single Pareto front, shown in (Figure 3(b)), is obtained, containing the dominating solutions for the various possible numbers of repairs, $N_{\text {rep }}$. Figure 3(c) shows the risk profiles associated with solution B1, selected since the associated total maintenance cost is the closest to the cost associated with solution A1, described in the previous section (Figure 2(b)). Solution B1 is characterized by two repair actions, as well, occurring at 39 and 62 years. In this case, first the deck is repaired, since it is associated with the highest component risk at 39 years, followed by essential maintenance on the exterior girders, having the highest associated risk at 62 years. In this case, the cost of the maintenance plan, evaluated by Eq. (3), is $\$ 69,631$. Figure 3(d) illustrates risk profiles obtained by the maintenance plan associated with solution B2, characterized by five maintenance actions. The deck is, in this case, repaired three times (at 24, 42 and 66 years), while the exterior and interior girders once (at 36 and 50 years, respectively), with a total maintenance cost of $\$ 201,706$.

Comparison between reliability-based and risk-based system maintenance optimizations

To compare the solutions obtained by the two previously described optimization formulations, it is convenient to plot the two Pareto fronts as total maintenance cost of each solution versus the maximum system annual failure probability $P_{s y s, \max }$ associated with each solution (Figure 4(a)). Solutions A1 and B1 have been selected, respectively, from the reliability-based and 
risk-based optimization results since they have, approximately, the same total maintenance costs (therefore, they are horizontally aligned in Figure 4(a)). By comparing the two solutions obtained for the considered bridge, it is observed that the solution associated with the riskbased optimal strategy has a lower maximum failure probability than that associated with the reliability-based strategy. This is due to the additional information contained implicitly into the annual risk (i.e. the consequences of the structural failure). Repair priority based on component risk has, indeed, higher cost-efficiency than priority based simply on component reliability.

In the bridge example the exterior girders are the components with the lowest reliability and, therefore, they are the first components to be repaired using the reliabilitybased approach. However, the effect of such repair on the system failure probability is not significant. On the contrary, the deck is the component with highest risk (i.e. highest repair priority in the risk-based approach) and also the most critical component (i.e. only series component in the system). For the same reasons, when considering solutions of the two Pareto fronts associated with the same maximum system failure probability (solutions A1 and B3 in Figure 4(a)), the risk-based maintenance plan has a higher cost-efficiency than the reliability-based one. Figure 4(b) reports the maintenance schedule corresponding to each of the three solutions considered, and Figure 4(c) the cumulative cost profiles.

While risk-based maintenance has certainly advantages in terms of cost-efficiency of the resulting solutions, evaluation of risk profiles for the optimization procedures involves a higher computational effort than that associated with reliability profiles. For the studied case, evaluation of reliability profiles requires the calculation of the component and system failure probabilities each year. On the other hand, evaluations of direct and indirect risks by Eqs. (8) additionally require the computation of the probabilities of only one component failing $P\left(F_{i} \bar{F}_{j \neq i}\right)$. By considering the bridge symmetry, this has to be computed three times, once for the deck, once for the exterior girders, and once for the interior girders. Then, for the exterior and interior girders cases, the failure probability of the system without the failed component $P\left(F_{s y s} \mid F_{i} \bar{F}_{j \neq i}\right)$ has to be calculated; in the case of deck failure this conditional probability is one. This procedure requires, therefore, the evaluation of the failure probability 
associated with five additional systems. Therefore, the computational time required for the risk-based optimization is about six times longer than that associated with the reliabilitybased optimization.

\section{Bi-objective optimization based on lifetime distributions and maintenance cost}

Lifetime distributions are representations of the performance state of the system over its lifecycle through functions that define the distribution of the system time to failure, considered as a continuous, non-negative random variable [Leemis 1995]. The distribution of the time to failure can be represented by closed-form expressions, depending on the structure and its loadings. The distribution of the system time to failure can be computed, if the type of system (i.e., series, parallel, series-parallel) and the lifetime distributions of its components are known.

Use of lifetime distributions for optimal maintenance planning has been already proposed [Yang et al. 2006b, Orcesi and Frangopol 2011]. The main advantage of lifetime distributions consists in dealing with closed-form expressions, while annual performance indicators require approximate numerical methods. Various lifetime distributions can be defined. In this paper the attention is focused on the availability and hazard functions.

The availability function (i.e. the probability of the system or component to be functional at each instant of time) is, by definition, particularly appropriate for maintenance oriented decision-making, since one of the main objectives of a maintenance plan is, indeed, to maximize the time of functionality of the system itself over its life-cycle. The availability function can also take into account the lack of functionality due to repair times [Ang and Tang 1984]. Multi-objective optimization for determining optimal essential and preventive maintenance schedules has been proposed in [Okasha and Frangopol 2010a].

The hazard function is, instead, related to the derivative of the availability with respect to time and, therefore, represents an index of how fast the system tends to become non-functional at each time. While the hazard function is extensively used in other scientific fields, applications to maintenance plan optimization for civil engineering structures are 
scarce. Preventive maintenance schedules through hazard function in civil structures are reported in [Caldeira Duarte et al. 2006, Baek et al. 2009]. Recently, the authors have proposed to use the hazard function for inspection/repair planning on single component systems by single-objective optimization [Barone and Frangopol 2013], and on multicomponent systems by multi-objective optimization considering also inspection errors [Barone et al. 2014].

\section{Availability function and total maintenance cost}

The system availability is defined as the probability of the system being functioning at a given instant of time [Ang and Tang 1984]. When no maintenance action is performed on the structure, the availability function $A(t)$ coincides, by definition, with the survivor function $S(t):$

$$
A(t)=S(t)=P\left[T_{F} \geq t\right]
$$

that is the probability that the system is still surviving (and therefore functioning as well) at a given time instant $t$. In Eq. (16), $T_{F}$ is the system failure time [Leemis 1995]. Contrary to the survivor function, that is a continuous, non-increasing function in time, the availability function increases its value whenever the system is repaired. In particular, under the assumption that the repair action returns the system to its original performance, its availability is restored to its original value (i.e., $A(t)=1$ ) after the repair. If $n$ consecutive essential maintenance actions are performed on a component at times $T_{1}, \ldots, T_{n}$, assuming that $T_{0}=0$ is the initial observation time, the survivor function $S_{i}(t)$ becomes:

$$
S_{i}(t)= \begin{cases}S_{i}(t) & t<T_{1} \\ S_{i}\left(t-T_{n}\right) \prod_{k=1}^{n} S_{i}\left(T_{k}-T_{k-1}\right) & T_{n} \leq t \leq T_{n+1}, k \geq 1\end{cases}
$$

$S_{i}(t)$ is continuous and non-increasing over time. The corresponding availability function is obtained from the survivor function as [Okasha and Frangopol 2010b]: 


$$
A_{i}(t)= \begin{cases}S_{i}(t) & t<T_{1} \\ S_{i}\left(t-T_{n}\right) & T_{n} \leq t \leq T_{n+1}, k \geq 1\end{cases}
$$

In the following, it is assumed that the times to failure of the bridge deck and girders follow the Weibull distribution:

$$
A_{i}(t)=S_{i}(t)=e^{-\left(\lambda_{i}\right)^{k_{i}}} \quad i=0, \ldots, 4
$$

where $\lambda_{0}=8 \cdot 10^{-3}$ and $k_{0}=2.4$ for the deck, $\lambda_{1}=\lambda_{4}=8 \cdot 10^{-3}$ and $k_{1}=k_{4}=2.3$ for the exterior girders, $\lambda_{2}=\lambda_{3}=6 \cdot 10^{-3}$ and $k_{2}=k_{3}=2.1$ for the interior girders. Under the assumption of statistically independent failure modes and considering the series-parallel model shown in Figure 1(b), the system availability is defined as:

$$
A_{s y s}(t)=S_{s y s}(t)=S_{0}(t) \prod_{i=1}^{3}\left(1-\left(1-S_{i}(t)\right)\left(1-S_{i+1}(t)\right)\right)
$$

where $S_{0}(t)$ is the survivor function associated with the bridge deck, and $S_{i}(t)(i=1, \ldots, 4)$ are the survivor functions associated with the bridge girders. System and component availability of the studied bridge superstructure are shown in Figure 5(a).

As indicated previously, it is necessary to establish a criterion for selecting which component should be repaired at each time. For the availability-based case, an availability importance factor is defined as:

$$
I A_{i}(t)=\frac{A_{s y s}^{(i)}(t)-A_{s y s}(t)}{A_{s y s}(t)}
$$

where $A_{s y s}^{(i)}(t)$ is the availability of the system after repairing the $i$-th component at the instant $t$, while $A_{\text {sys }}(t)$ is the availability of the same system before the repair. Since after the repair the system availability is not decreasing, $I A_{i}(t) \geq 0$. Therefore, this importance factor is an index of the gain in availability obtained by repairing the associated component. Moreover, a 
normalized importance factor $I A_{i}^{0}(t)$, assuming values in the interval $[0 ; 1]$, can be defined as follows:

$$
I A_{i}^{0}(t)=\frac{I A_{i}(t)}{\sum_{i=0}^{4} I A_{i}(t)}
$$

In the following, at each maintenance time, the component with the maximum normalized importance factor $I A_{i}^{0}$ at the repair instant (i.e. the component that, when repaired, returns the maximum gain in availability) is selected for repair.

In this section, the maintenance optimization problem is defined in terms of the availability function. In particular, maintaining the same constraints and repair costs defined in the previous sections, and considering the total maintenance cost as second objective, the bi-objective optimization problem is reformulated to maximize the minimum value of the system availability over the system life-cycle. Similarly to the reliability-based case, the inverse of the minimum availability is considered, so that a minimization problem is defined for both objectives:

Given: $A_{i}(t), C_{i}, r \quad i=0, \ldots, 4$

Find: $N_{\text {rep }}, \mathbf{t}_{\text {rep }}=\left\{t_{1}, \ldots, t_{N_{\text {rep }}}\right\}$

To minimize: $\left\{\begin{array}{l}\left(A_{\text {min }}(t)\right)^{-1} \\ C_{\text {tot }}\end{array} \quad 0 \leq t \leq 75\right.$ years

Such that: $\left\{\begin{array}{l}t_{j}-t_{j-1} \geq 6 \text { years } \\ t_{N_{\text {rep }}} \leq 66 \text { years }\end{array} \quad j=1, \ldots, N_{\text {rep }}\right.$ 
where $A_{i}(t)$ are the availability functions associated with the bridge components, and $A_{\text {min }}(t)$ is the minimum value assumed by the system availability function over the considered time span.

By using genetic algorithms, as described in the previous sections, the Pareto front illustrated in Figure 5(b) is obtained. Also in this case, discontinuities in the Pareto front corresponds to change in the number of repairs $N_{\text {rep }}$. System and components availability profiles of a selected solution (solution C), are shown in Figure 5(c). Solution C belongs to the subset of solutions involving three maintenance actions, among the solutions in the availability-based Pareto front. In particular, the maintenance plan is constituted by repair of the deck after 29 years, followed by repair of the interior girders at the year 47, and finally a new essential maintenance performed on the deck at the year 55. The selected repaired components are associated with the maximum gain in availability at the corresponding repair times. The total maintenance cost associated with solution C is $\$ 121,503$.

\section{Hazard function and total maintenance cost}

The hazard function is defined as the ratio between the failure probability in the time interval $[t, t+\Delta t]$ conditioned by the system still surviving at time $t$, and the time interval $\Delta t$ itself, with $\Delta t \rightarrow 0$, that is:

$$
h(t)=\lim _{\Delta t \rightarrow 0} \frac{P\left[t \leq T_{f} \leq t+\Delta t \mid T_{f} \geq t\right]}{\Delta t}=-\frac{S^{\prime}(t)}{S(t)}
$$

As shown in Eq. (27), the hazard function is related to the derivative of the survivor function over time, $S^{\prime}(t)$. Therefore, instead of providing a direct indication on the functioning state of the system, the hazard function represents the rate of failure of the system itself, i.e. how fast the system tends to fail at a given instant.

Maintaining the same assumption of Weibull distribution for the time to failure of each bridge component, the hazard functions associated with deck and girders are defined as: 


$$
h_{i}(t)=k_{i} \lambda_{i}^{k_{i}} t^{k_{i}-1} \quad i=0, \ldots, 4
$$

where the value of $\lambda_{i}$ and $k_{i}$ for deck and girders have been defined previously (i.e., Eq. (19)). Moreover, under the assumption of statistically independent failure modes, the hazard function for the series-parallel system shown in Figure 1(b) can be obtained as:

$$
h_{s y s}=h_{0}+\sum_{i=1}^{3} \frac{h_{i}\left(1-S_{i+1}\right)+h_{i+1}\left(1-S_{i}\right)}{1-\left(1-S_{i}\right)\left(1-S_{i+1}\right)}
$$

where $h_{0}(t)$ is the hazard function associated with the bridge deck, and $h_{i}(t)$ and $S_{i}(t)$ are hazard and survivor functions associated with the girders, respectively. System and components hazard functions for the studied bridge superstructure are shown in Figure 6(a).

A hazard importance factor indicating the reduction in the hazard system due to repair is introduced as:

$$
I h_{i}(t)=\frac{h_{s y s}(t)-h_{s y s}^{(i)}(t)}{h_{s y s}(t)}
$$

where $h_{\text {sys }}^{(i)}(t)$ is the system hazard function after performing essential maintenance on the $i$-th component at instant $t$, while $h_{s y s}(t)$ is the system hazard function before the maintenance is performed. Since the system hazard function is not increasing after repair of a component, $I h_{i}(t) \geq 0$. In this case, a normalized hazard importance factor is considered as:

$$
I h_{i}^{0}(t)=\frac{I h_{i}(t)}{\sum_{i=0}^{4} I h_{i}(t)}
$$

and repair priority has been given to the component with the highest $I h_{i}^{0}$.

The maintenance optimization problem in terms of hazard and total maintenance cost has been defined taking into account the constraints with respect to the maintenance times, as 
seen in the previous sections, and using the same repair costs and discount rate of money, as follows:

Given: $S_{i}(t), h_{i}(t), C_{i}, r \quad i=0, \ldots, 4$

Find: $N_{\text {rep }}, \mathbf{t}_{\text {rep }}=\left\{t_{1}, \ldots, t_{N_{\text {rep }}}\right\}$

To minimize: $\left\{\begin{array}{l}h_{\max }(t) \\ C_{t o t}\end{array} \quad 0 \leq t \leq 75\right.$ years

Such that: $\left\{\begin{array}{l}t_{j}-t_{j-1} \geq 6 \text { years } \\ t_{N_{\text {rep }}} \leq 66 \text { years }\end{array} \quad j=1, \ldots, N_{\text {rep }}\right.$

where $S_{i}(t)$ and $h_{i}(t)$ are the survivor and hazard function associated with the ith bridge components, and $h_{\max }(t)$ is the maximum value of the system hazard function over the observed time window. Therefore, analogously to the risk-based approach (where the maximum value of risk was minimized), in this case the first goal is to minimize the maximum value of the system hazard. The Pareto front of optimal solutions obtained by genetic algorithm optimization is shown in Figure 6(b), while system and components hazard profiles for solution D are shown in Figure 6(c). The latter solution is associated with a maintenance plan involving four maintenance actions: essential maintenance of the deck at 29 and 53 years, and essential maintenance of the interior girders at 44 and 64 years, respectively. The total cost of the maintenance plan is $\$ 147,318$.

Comparison between availability-based and hazard-based system maintenance optimizations With the aim of comparing the two sets of solutions obtained by the availability and hazardbased system optimization approaches, the associated Pareto fronts have been plotted in Figure 7(a) considering the total maintenance cost as a function of the minimum system availability associated to each solution; on the other hand, Figure 7(b) shows the same set of 
solutions in terms of total maintenance cost and maximum hazard associated with each solution. The two Pareto fronts are very similar when the number of repair is low (solutions associated with low maintenance costs). Solutions C and D, shown in Figure 7(c) and 7(d), respectively, have been selected so that the same minimum system availability is obtained in the two cases. However, solution C, obtained using the availability-based approach, requires a fewer number of repairs $\left(N_{\text {rep }}^{C}=3\right.$ ), and, therefore, it is associated with less maintenance cost than solution D, resulting from the hazard-based approach $\left(N_{\text {rep }}^{D}=4\right)$. This should be expected, since solutions that are dominant in one approach do not necessarily belong to the Pareto front obtained from the other approach. This is evident in Figure 7(b) where it can be observed that some availability-based solutions having high total maintenance cost are less cost-efficient than other solutions obtained with the hazard-based approach. Figures 7(c) and (d) show the system availability and system hazard profiles, respectively, associated with the solutions $\mathrm{C}$ and $\mathrm{D}$. It is worth noting that the availability, hazard, and the remaining lifetime distribution functions contain the same level of information on the system performance. However, the two presented optimization procedures aim at controlling different aspects of the life-cycle maintenance problem: (a) the probability that the system is functional when availability is selected as objective of the optimization, and (b) the rate of failure of the system when the hazard function is selected as objective of the optimization.

The difference between availability-based and hazard-based approaches in the time required for the computation is negligible. In both cases, all expressions required for the calculation are defined in analytical form, making the computational process extremely fast. This is the main advantage that emerges when comparing lifetime distributions to annual performance indicators. The four optimization formulations have all been implemented running on a single core of a 64-bit, 2.33GHz Intel(R) Xeon(R) CPU E5410. The computational times required for the reliability-based approach is nine hours and 52 minutes, while only 17 minutes are necessary to complete the optimization considering availability or hazard functions. On the other hand, lifetime distributions are defined in analytical forms only for specific cases of correlation among the system components (in general, statistically independent or perfectly correlated failure modes). Therefore, all information regarding the 
actual correlations among the random variables involved in the definition of components and system limit states is lost. Consequently, solutions provided by using annual performance indicators, although obtained through approximate numerical procedures, should be considered more representative of the actual system performance over the structure life-cycle.

\section{Conclusions}

In this paper, the optimization of life-cycle maintenance plans of deteriorating structures has been considered using four different bi-objective approaches. In the first two cases, annual performance indicators are considered, in particular annual reliability index and annual risk. Then, lifetime distributions approaches are used by considering availability and hazard functions as goals of the optimization problem. In all cases, the second goal of the optimization is the minimization of the total cost of the maintenance plan. Results of the four proposed approaches are illustrated through a numerical example relative to an existing bridge superstructure, and the advantages and drawbacks of the four approaches are compared and discussed. The following conclusions are drawn:

1. Pareto fronts obtained by using reliability and risk-based approaches are extremely different, although the same constraints and repair costs have been considered, and the same optimization method has been used. Considering the maximum system failure probability reached over the system life-cycle, riskbased optimum maintenance plans are more cost-efficient than their reliability-based counterparts. Therefore, involving consequences of the component and system failures into the decision-making process plays a crucial role in the determination of optimal maintenance plan.

2. The risk-based approach requires not only an initial estimation of direct and indirect failure consequences, but also the evaluation of several additional probabilities of failure of single components and conditional probabilities of failure. This process increases the computational effort required to perform the optimization. The magnitude of this increase is proportional to the number of components of the system. 
3. Availability-based and hazard-based optimization formulations may result in similar solutions. In particular, several optimal solutions are identical when considering less than four repairs. However, when the number of maintenance actions is increased, optimal solutions obtained by availability and hazardbased approaches are different.

4. Computational efforts required for the availability and hazard-based approaches are several times smaller than those associated with the reliability and risk-based approaches. However, information regarding actual correlations among random variables involved in the limit states is approximated by considering upper and lower correlation bounds. For this reason, annual performance indicators, although requiring the use of approximate numerical methods, have to be considered more reliable indicators than those associated with lifetime distributions.

\section{Acknowledgments}

The support by grants from (a) the National Science Foundation (NSF) Award CMS-0639428, (b) the Commonwealth of Pennsylvania, Department of Community and Economic Development, through the Pennsylvania Infrastructure Technology Alliance (PITA), (c) the U.S. Federal Highway Administration (FHWA) Cooperative Agreement Award DTFH61-07H-00040, (d) the U.S. Office of Naval Research (ONR) Awards Number N00014-08-1-0188 and Number N00014-12-1-0023, (e) the National Aeronautics and Space Administration (NASA) Award NNX10AJ20G is gratefully acknowledged. The opinions presented in this paper are those of the authors and do not necessarily reflect the views of the sponsoring organizations.

\section{References}

Ang, A.H-S., De Leon, D. (2005), "Modeling and analysis of uncertainties for risk-informed decisions in infrastructures engineering”, Structure and Infrastructure Engineering, 1(1):1931. 
Ang, A.H-S., Tang, W.H. (1984), "Probability concepts in engineering planning and design. Volume II - Decision, risk and reliability”, John Wiley and Sons, New York.

Akgül, F. (2002), "Lifetime system reliability prediction for multiple structure types in a bridge network”, PhD Thesis, Department of Civil, Environmental, and Architectural Engineering, University of Colorado, Boulder, Colorado.

Baek G., Kim K., Kim S. (2009), “Optimal preventive maintenance inspection period on reliability improvement with Bayesian network and hazard function in gantry crane”, Proceedings of the $6^{\text {th }}$ International Symposium on Neural Networks: Advances in Neural Networks - Part III, Wuhan, China, Springer-Verlag, 1190-1196.

Barone, G., Frangopol, D.M. (2013), "Hazard-based optimum lifetime inspection/repair planning for deteriorating structures”, Journal of Structural Engineering, 139(12), 1-12.

Barone, G., Frangopol, D.M. (2014), "Reliability, risk and lifetime distributions as performance indicators for life-cycle maintenance of deteriorating structures”, Reliability Engineering and System Safety, 123, 21-37.

Barone, G., Frangopol, D.M., Soliman M. (2014), “Optimization of life-cycle maintenance of deteriorating bridges considering expected annual system failure rate and expected cumulative cost”, Journal of Structural Engineering, 140(2), 1-13.

Bocchini P., Frangopol D.M. (2011), “A probabilistic computational framework for bridge network optimal maintenance scheduling”, Reliability Engineering and System Safety, 96, 332-349. 
Caldeira Duarte J.A., Craveiro J.C.T.A., Trigo T.P. (2006), “Opmitization of the preventive maintenance plan of a series components system”, International Journal of Pressure Vessels and Piping, 83, 244-248.

Deb, K. (2001), “Multi-objective optimization using evolutionary algorithms”, John Wiley and Sons, New York.

Ellingwood, B.R. (2005), “Risk-informed condition assessment of civil infrastructure: state of practice and research issues”, Structure and Infrastructure Engineering, 1(1), 7-18.

Estes, A.C., Frangopol, D.M. (1998), "RELSYS: A computer program for structural system reliability analysis”, Structural Engineering Mechanics, 6(8), 901-919.

Frangopol, D.M., Lin K.Y., Estes A.C. (1997), “Life-cycle cost design of deteriorating structures”, Journal of Structural Engineering, 123:1390-1401.

Frangopol, D.M. (2011), "Life-cycle performance, management and optimisation of structural systems under uncertainty: accomplishments and challenges”, Structure and Infrastructure Engineering, 7(6), 389-413.

Leemis, L.M. (1995), “Reliability, probabilistic models and statistical methods”, Prentice Hall, New Jersey.

Marsh, P.S., Frangopol, D.M., (2008), "Reinforced concrete bridge deck reliability model incorporating temporal and spatial variations of probabilistic corrosion rate sensor data”, Reliability Engineering and System Safety, 93, 394-409.

Moan, T., (2005), "Reliability-based management of inspection, maintenance and repair of offshore structures”, Structure and Infrastructure Engineering, 1(1), 33-62. 
Okasha, N.M., Frangopol, D.M. (2010a), "Redundancy of structural systems with and without maintenance: an approach based on lifetime functions”, Reliability Engineering and System Safety, 95, 520-533.

Okasha, N.M., Frangopol, D.M. (2010b), “Novel approach for multicriteria optimization of life-cycle preventive and essential maintenance of deteriorating structures”, Journal of Structural Engineering, 138(8), 1009-1022.

Orcesi, A.D., Frangopol, D.M. (2011), "Use of lifetime functions in the optimization of nondestructive inspection strategies for bridges”, Journal of Structural Engineering, 137(4), 531-539.

Saydam, D., Frangopol, D.M. (2011), “Time-dependent performance indicators of damaged bridge superstructures”, Engineering Structures, 33, 2458-2471.

Stein, S.M., Young, G.K., Trent, R.E., Pearson, D.R. (1999), "Prioritizing scour vulnerable bridges using risk”, Journal of Infrastructure Systems, 5(3), 95-101.

Yang, S., Frangopol, D.M., Neves, L.C. (2006b), “Optimum maintenance strategy for deteriorating bridge structures based on lifetime functions”, Engineering Structures, 28, 196206.

Yang, S., Kawakami, Y., Frangopol, D.M., Neves, L.C. (2006a), "Use of lifetime functions in the optimization of interventions on existing bridges considering maintenance and failure costs”, Reliability Engineering and System Safety, 91(6), 698-705.

Wen, Y.K., Kang, Y.J. (2001), "Minimum building life-cycle cost design criteria. I. Methodology, and II. Applications”, Journal of Structural Engineering, 127,330-346. 
Zhu, B., Frangopol, D.M. (2013), "Risk-based approach for optimum maintenance of bridges under traffic and earthquake loads”, Journal of Structural Engineering, 139(3), 422-434. 


\section{Figure captions}

Figure 1 Bridge E-17-HS superstructure: (a) cross-section (adapted from Akgül 2002); (b) series-parallel model; (c) component and system annual failure probability profiles.

Figure 2 (a) Annual reliability index profile of the bridge superstructure; (b) reliabilitybased Pareto front; annual reliability index profile associated with solutions (c) A1 and (d) A2.

Figure 3 (a) Annual risk profile of the bridge superstructure; (b) risk-based approach Pareto front; annual risk profile associated with solutions (c) B1 and (d) B2.

Figure 4 (a) Comparison between reliability and risk-based Pareto fronts in terms of maximum annual system failure probability and total maintenance cost; (b) maintenance schedule associated with solutions A1, B1 and B3; and (c) cumulative cost profiles of solutions A1, B1 and B3.

Figure 5 (a) Availability of the bridge superstructure; (b) availability-based approach Pareto front; (c) availability associated with solution C.

Figure 6 (a) Hazard of the bridge superstructure; (b) hazard-based approach Pareto front; (c) hazard associated with solution D.

Figure 7 Comparison between availability and hazard-based Pareto fronts in terms of total maintenance costs and (a) minimum system availability and (b) maximum system hazard; (c) system availability for solutions C and D and (d) system hazard for solutions C and D. 
(a)

$9.144 \mathrm{~m}$
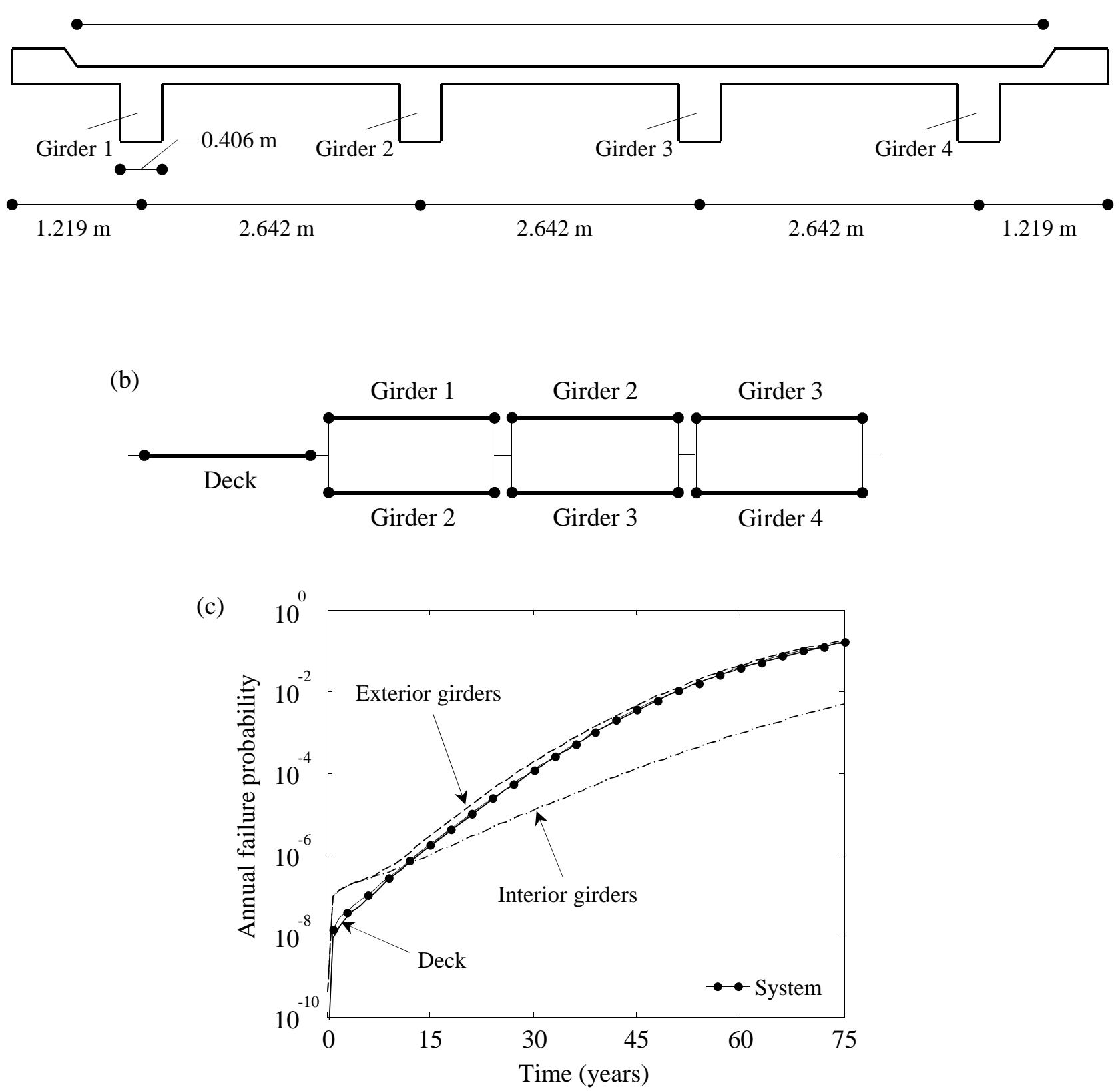

FIGURE 1 
(a)

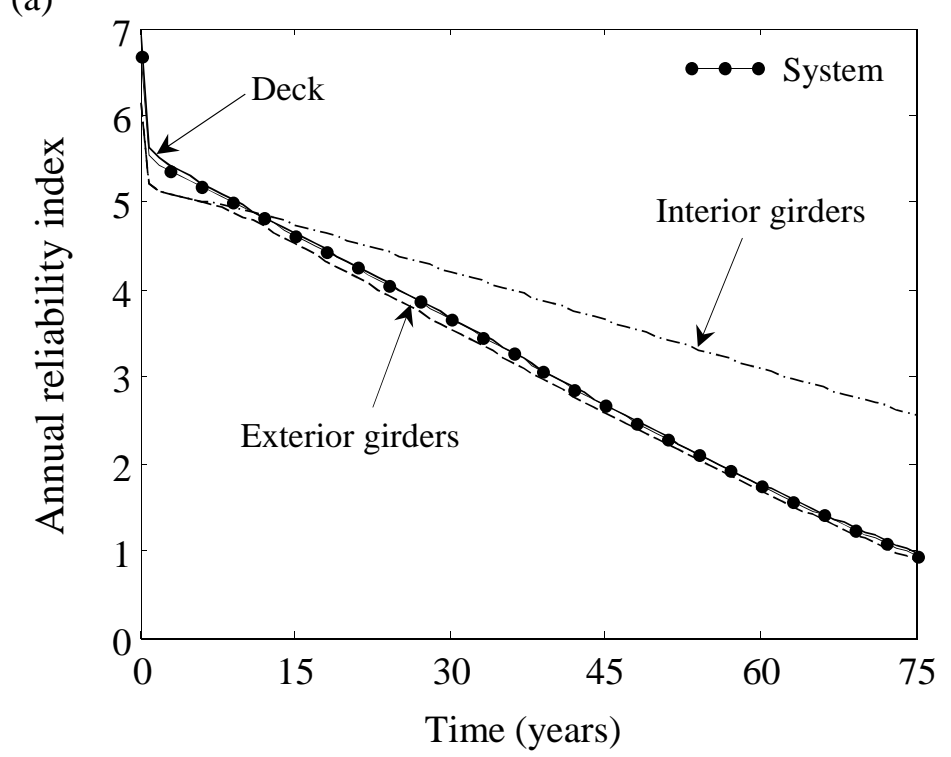

(c)

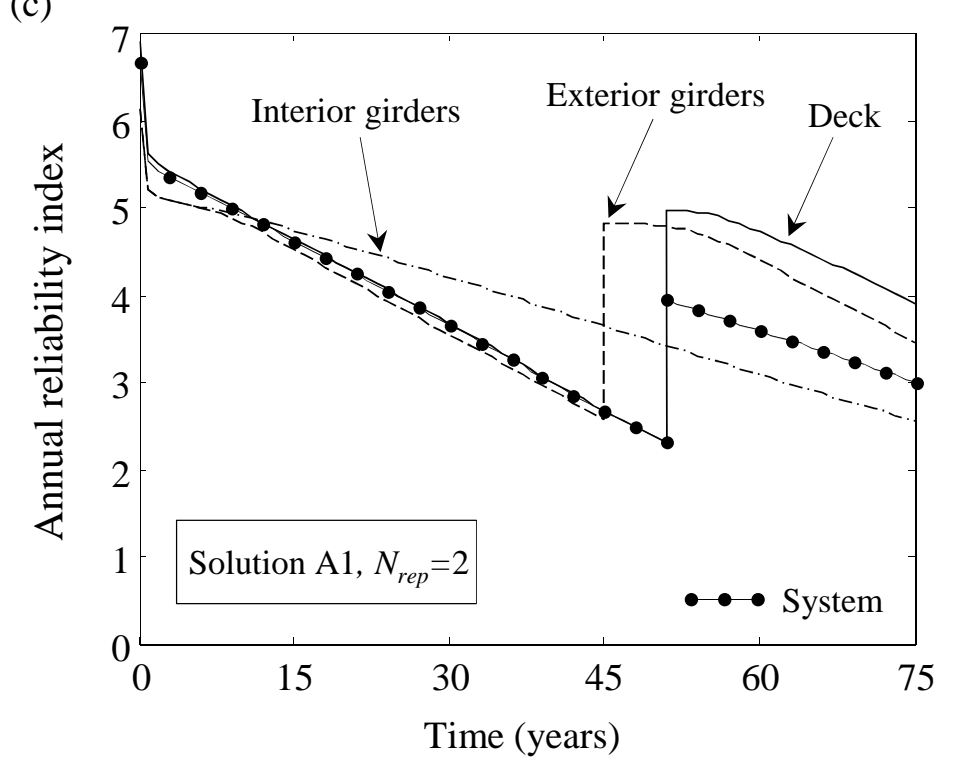

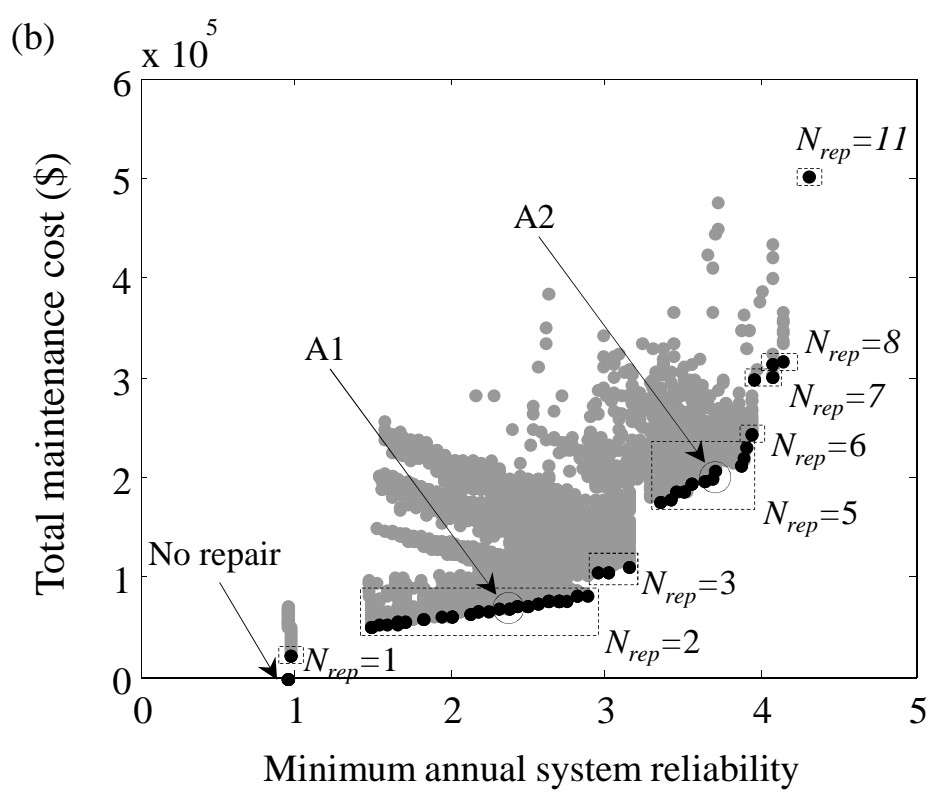

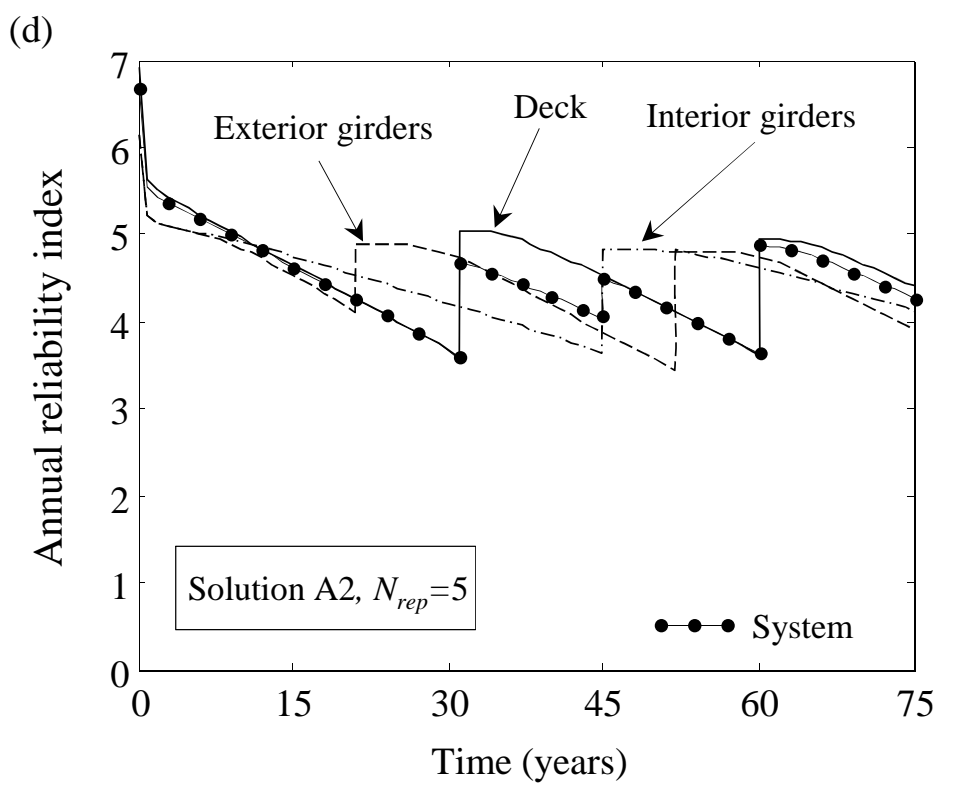

FIGURE 2 
(a)

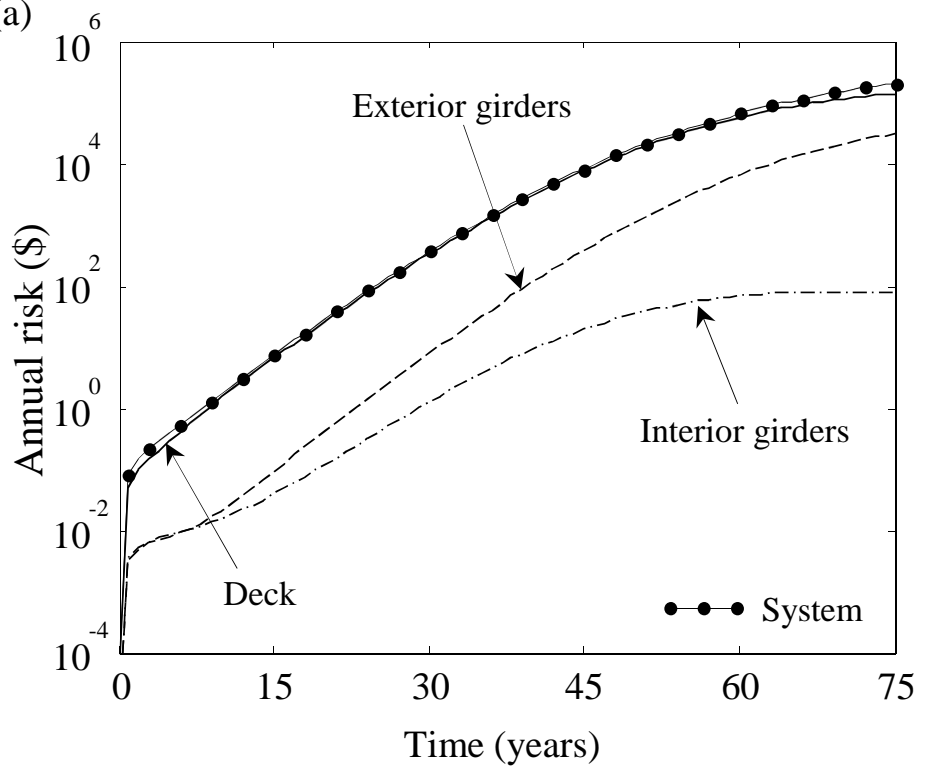

(c)

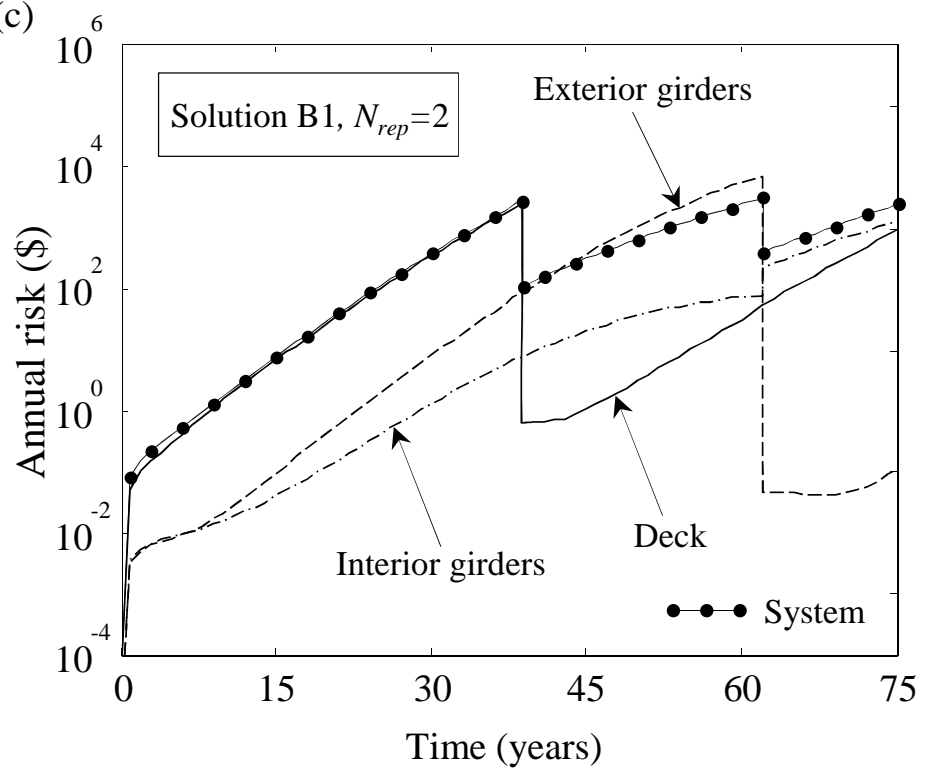

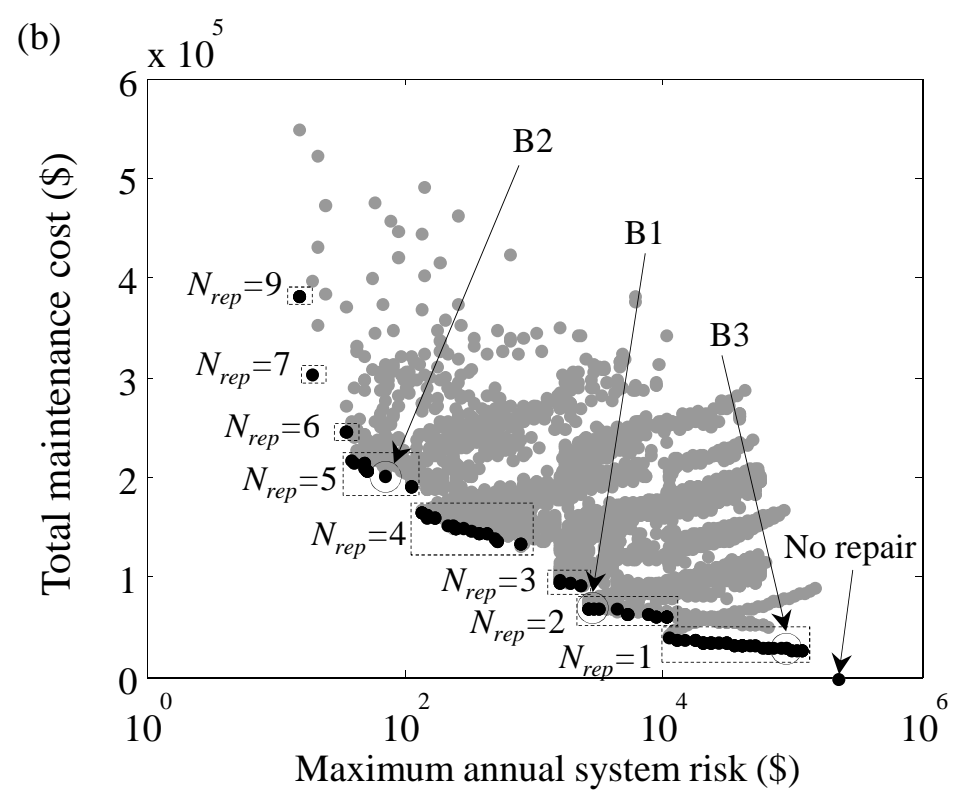

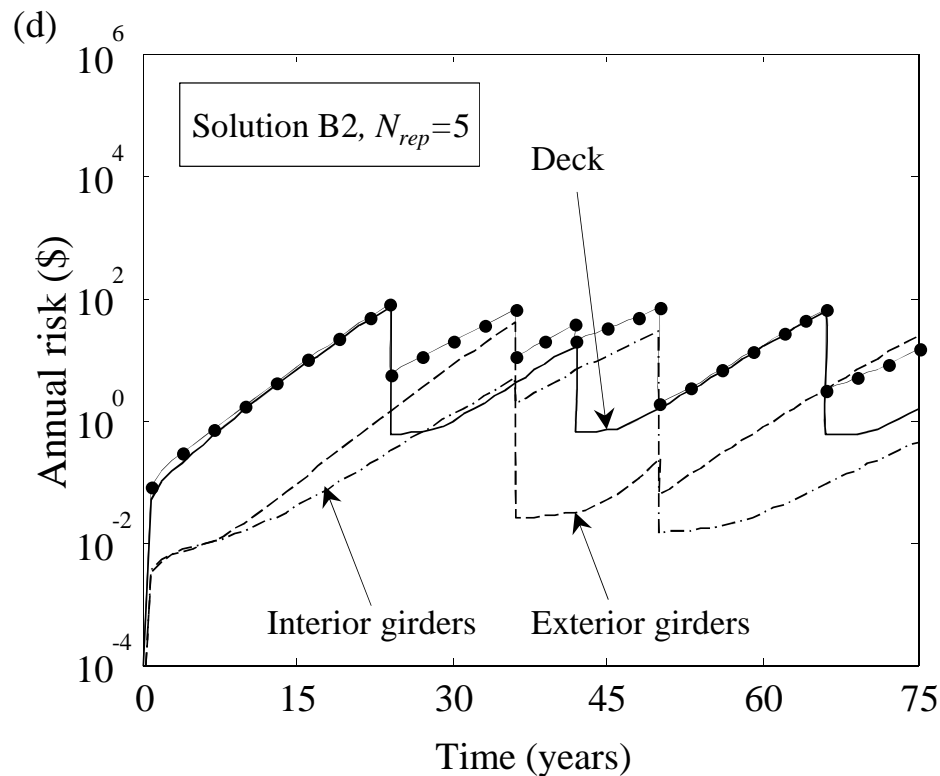

FIGURE 3 


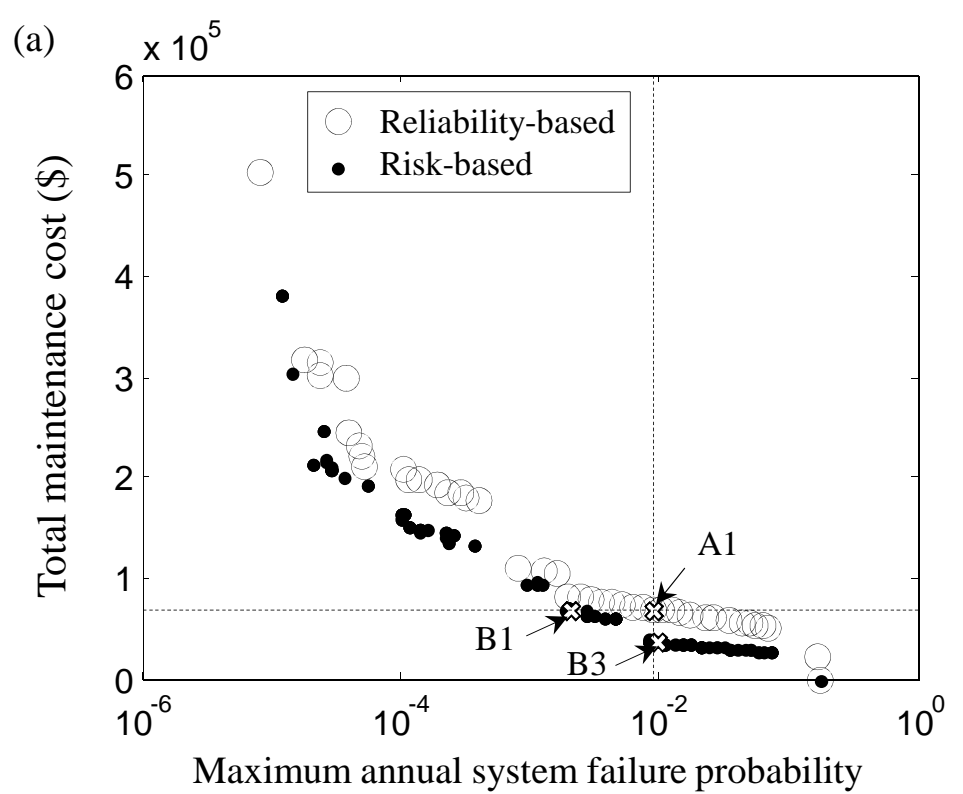

(b)

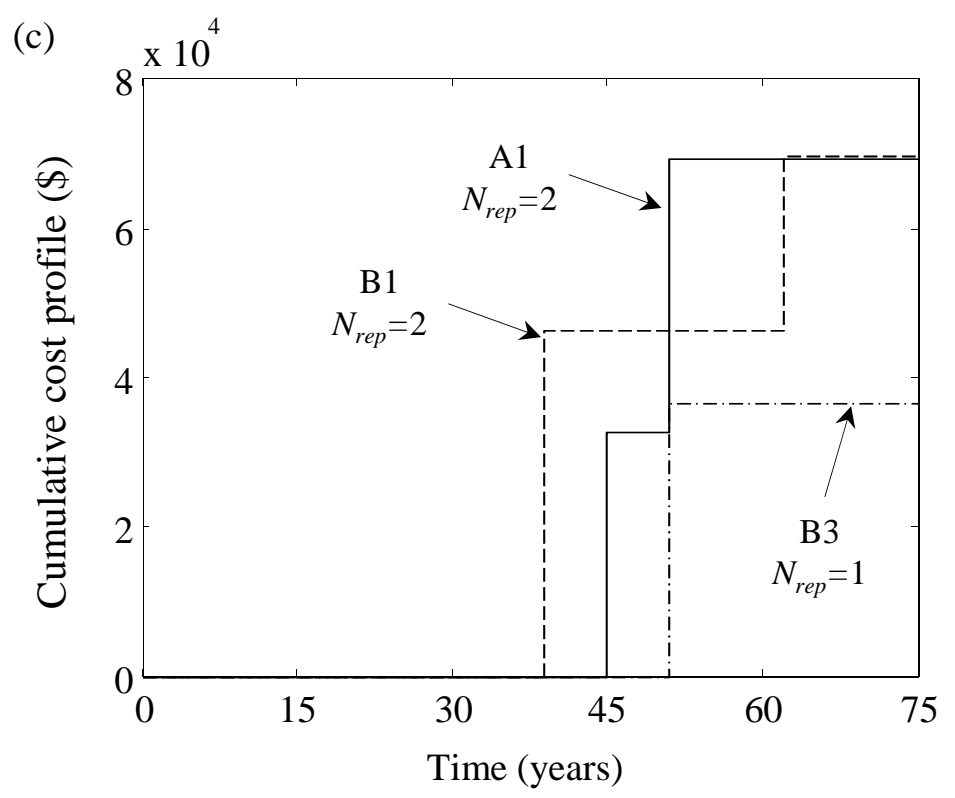

FIGURE 4 

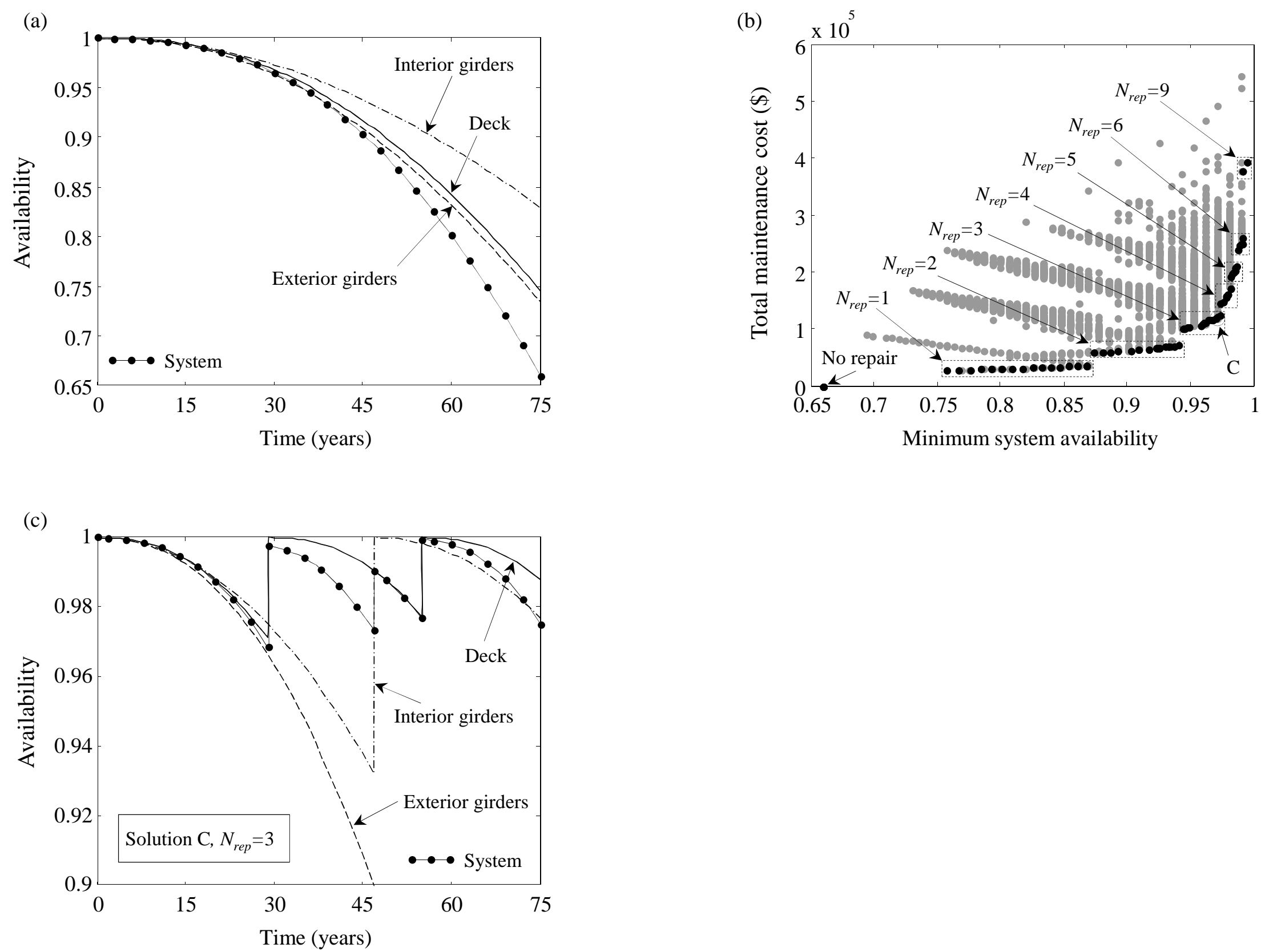

FIGURE 5 

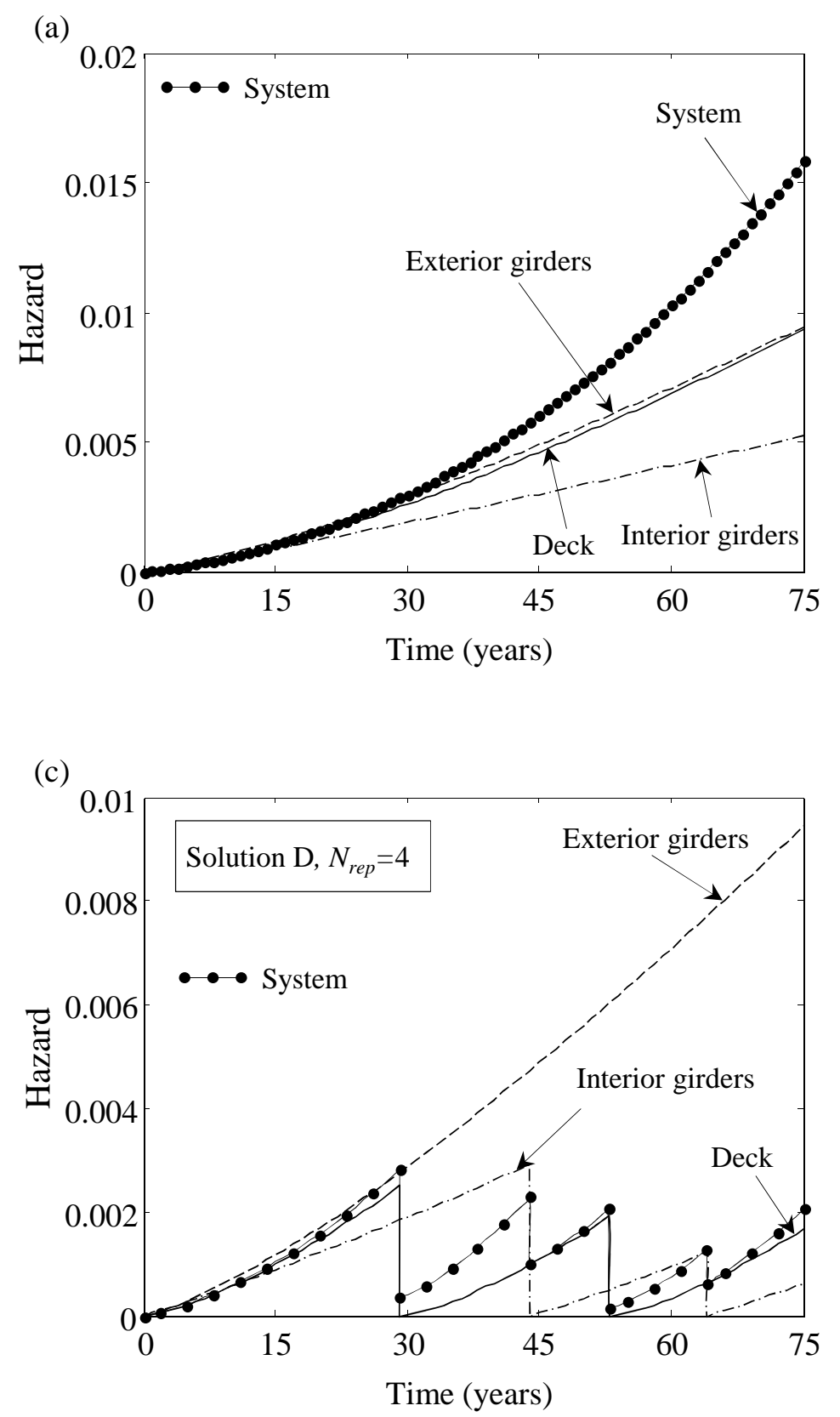

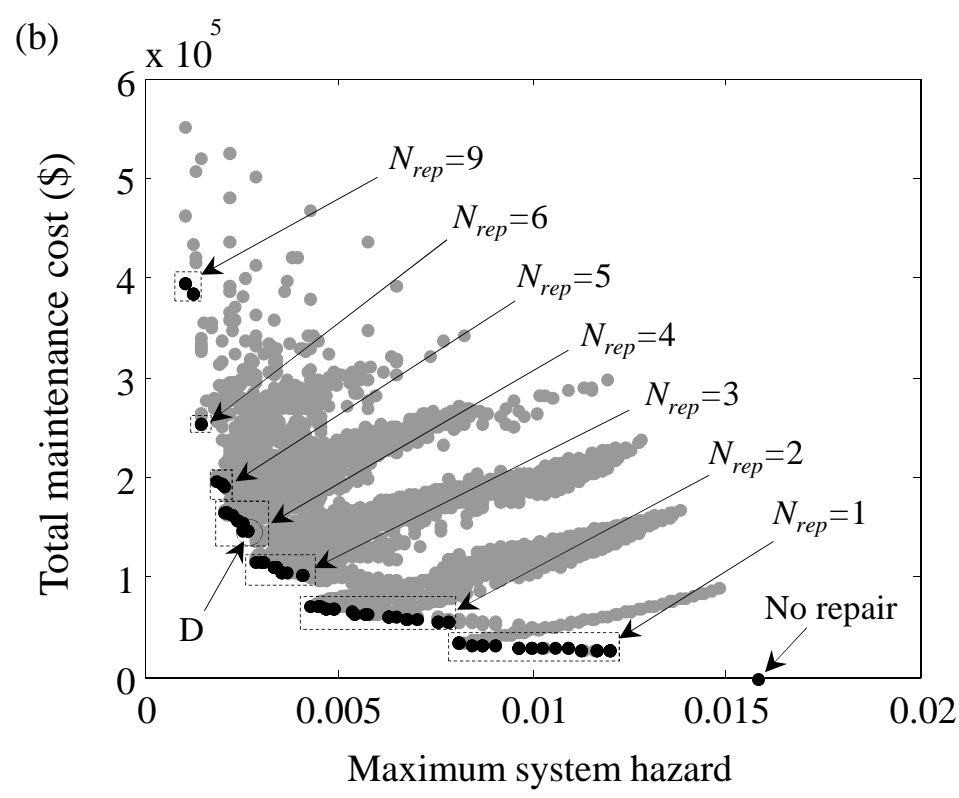

FIGURE 6 

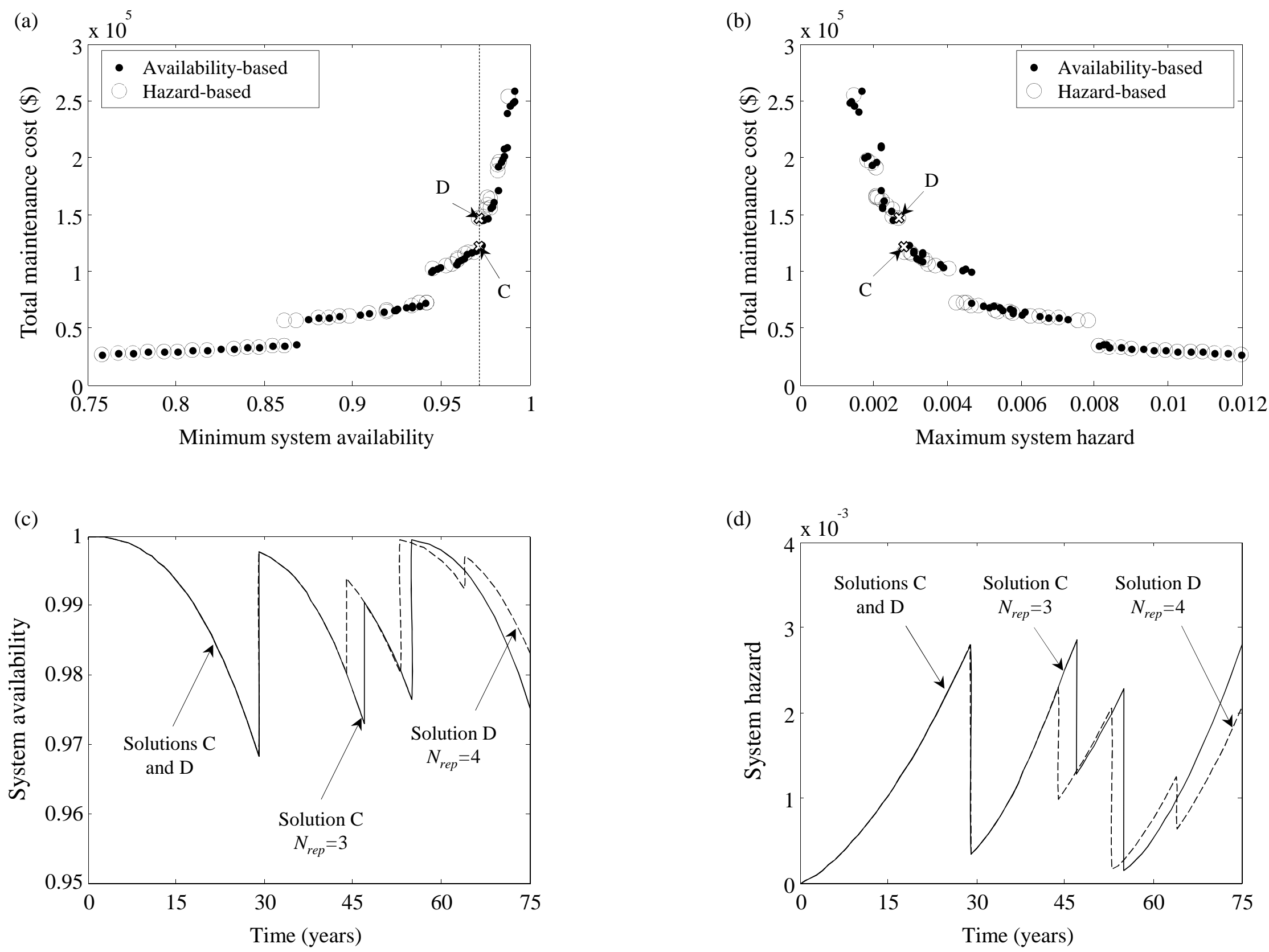

FIGURE 7 